DOI 10.4171/JEMS/193

Antonio Cañada $\cdot$ Salvador Villegas

\title{
Lyapunov inequalities for Neumann boundary conditions at higher eigenvalues
}

Received December 21, 2007

\begin{abstract}
This paper is devoted to the study of Lyapunov-type inequalities for Neumann boundary conditions at higher eigenvalues. Our main result is derived from a detailed analysis of the number and distribution of zeros of nontrivial solutions and their first derivatives, together with the use of suitable minimization problems. This method of proof yields new information on Lyapunov constants. For instance, we prove that as in the classical result by Lyapunov, the best constant is not attained. Additionally, we exploit the relation between Neumann boundary conditions and disfocality to provide new nonresonance conditions at higher eigenvalues.
\end{abstract}

Keywords. Neumann boundary value problems, resonance, higher eigenvalues, Lyapunov inequalities, existence and uniqueness

\section{Introduction}

The classical $L^{1}$ Lyapunov inequality for the Neumann boundary problem

$$
u^{\prime \prime}(x)+a(x) u(x)=0, \quad x \in(0, L), \quad u^{\prime}(0)=u^{\prime}(L)=0
$$

states that if

$$
a \in L^{1}(0, L) \backslash\{0\}, \quad \int_{0}^{L} a(x) d x \geq 0,
$$

is such that 1.1 has nontrivial solutions, then $\int_{0}^{L} a^{+}(x) d x>4 / L$, where $a^{+}(x)=$ $\max \{a(x), 0\}([5],[13])$. In [1] and [14] the authors generalize this result by providing, for each $p$ with $1 \leq p \leq \infty$, optimal necessary conditions for the boundary value problem 1.1 to have nontrivial solutions, given in terms of the $L^{p}$ norm of the function $a^{+}$. In particular, if $p=\infty$, it is proved that (1.1) has only the trivial solution if the function $a$ satisfies

$$
a \in L^{\infty}(0, L) \backslash\{0\}, \quad \int_{0}^{L} a \geq 0, \quad a^{+} \prec \pi^{2} / L^{2},
$$

where for $c, d \in L^{1}(0, L)$, we write $c \prec d$ if $c(x) \leq d(x)$ for a.e. $x \in[0, L]$ and $c(x)<d(x)$ on a set of positive measure. This is a very well known result which is usually

A. Cañada, S. Villegas: Departamento de Análisis Matemático, Universidad de Granada, 18071 Granada, Spain; e-mail: acanada@ugr.es, svillega@ugr.es

Mathematics Subject Classification (2010): 34B15, 34B05 
called the nonuniform nonresonance condition with respect to the first two eigenvalues $\lambda_{0}=0$ and $\lambda_{1}=\pi^{2} / L^{2}$ of the eigenvalue problem

$$
u^{\prime \prime}(x)+\lambda u(x)=0, \quad x \in(0, L), \quad u^{\prime}(0)=u^{\prime}(L)=0
$$

(see [8], [9] and [11]). From this point of view, it may be stated that the nonuniform nonresonance condition $(1.3)$ is in fact the $L^{\infty}$ Lyapunov inequality at the first two eigenvalues $\lambda_{0}$ and $\lambda_{1}$.

On the other hand, the set of eigenvalues of 1.4 is given by $\lambda_{n}=n^{2} \pi^{2} / L^{2}, n \in$ $\mathbb{N} \cup\{0\}$, and by using a general result due to Dolph [4], it can be proved that if for some $n \geq 1$ the function $a$ satisfies

$$
\lambda_{n} \prec a \prec \lambda_{n+1}
$$

then (1.1) has only the trivial solution (see [10, Lemma 2.1] for some generalizations of (1.5) to more general boundary value problems). It is clear that condition (1.5) cannot be obtained from the $L^{p}$ Lyapunov inequalities given in [1] and [14].

The previous observations motivate this article where, for any given natural number $n \geq 1$ and function $a$ satisfying $\lambda_{n} \prec a$, we obtain the $L^{1}$ Lyapunov inequality (the case of $L^{p}$ with $1<p<\infty$ has some special features and will be considered in a forthcoming paper). In particular, we prove that, as in the classical Lyapunov inequality, the best constant is not attained for any value of $n$. To the best of our knowledge this result is new if $n \geq 1$. In the $L^{\infty}$ case, the Lyapunov inequality is exactly 1.5 and in this sense, it is natural to say that this paper deals with Lyapunov inequalities at higher eigenvalues.

One of the main results of our paper is given by Lemma 2.2. below where we discuss in detail the number and distribution of zeros of $u$ and $u^{\prime}$, where $u$ is any nontrivial solution of the linear boundary value problem 1.1 .

In the second section we study the $L$ Lyapunov inequality when $\lambda_{n} \prec a$. The case where $a$ satisfies $A \leq a(x) \leq B$ a.e. in $(0, L)$, where $\lambda_{k}<A<\lambda_{k+1} \leq B$ for some $k \in \mathbb{N} \cup\{0\}$, has been considered in [6]. In that paper the authors use optimal control theory methods, specially Pontryagin's maximum principle.

In the last section we use the natural relation between Neumann boundary conditions and disfocality, given by Lemma 2.2. to obtain new results on the existence and uniqueness of solutions for linear resonant problems with Neumann boundary conditions. We use $L^{1}$ and $L^{\infty}$ Lyapunov constants. For example, by using Lemma 2.2 and the $L^{\infty}$ Lyapunov inequality, we can prove (Theorem 3.1) that if $a \in L^{\infty}(0, L), \lambda_{n} \prec a$ and there exists $0=y_{0}<y_{1}<\cdots<y_{2 n+1}<y_{2 n+2}=L$ such that

$$
\max _{0 \leq i \leq 2 n+1}\left\{\left(y_{i+1}-y_{i}\right)^{2}\|a\|_{L^{\infty}\left(y_{i}, y_{i+1}\right)}\right\} \leq \pi^{2} / 4
$$

and, in addition, $a$ is not the constant $\pi^{2} / 4\left(y_{i+1}-y_{i}\right)^{2}$ at least in one of the intervals $\left[y_{i}, y_{i+1}\right], 0 \leq i \leq 2 n+1$, then 1.1 has only the trivial solution (this kind of functions $a$ are usually named $2(n+1)$-step potentials).

The above hypothesis is optimal in the sense that if $a$ is the constant $\pi^{2} / 4\left(y_{i+1}-y_{i}\right)^{2}$ in each of the intervals $\left(y_{i}, y_{i+1}\right), 0 \leq i \leq 2 n+1$, then 1.1 has nontrivial solutions (see Remark7 7 in Section 3). If $y_{i}=i L / 2(n+1), 0 \leq i \leq 2 n+2$, we have the so called 
nonuniform nonresonance conditions at higher eigenvalues ([4], [10]) but if for instance $y_{j+1}-y_{j}<L / 2(n+1)$ for some $j, 0 \leq j \leq 2 n+1$, then $a$ can satisfy $\|a\|_{L^{\infty}\left(y_{j}, y_{j+1}\right)}=$ $\pi^{2} / 4\left(y_{j+1}-y_{j}\right)^{2}$ (which is a quantity greater than $\left.\lambda_{n+1}=(n+1)^{2} \pi^{2} / L^{2}\right)$ as long as $a$ satisfies (1.6) for each $i \neq j$.

Additionally, as has been done in [1], [2], [3] and [6], the linear study can be combined with the Schauder fixed point theorem to provide new conditions for the existence and uniqueness of solutions for resonant nonlinear problems (Theorem 3.3. Also, we may deal with other boundary value problems. Finally, one can expect that some results hold true in the case of the Neumann boundary value problem for partial differential equations

$$
\Delta u(x)+a(x) u(x)=0, \quad x \in \Omega, \quad \frac{\partial u(x)}{\partial n}=0, \quad x \in \partial \Omega,
$$

where $\Omega$ is a bounded and regular domain in $\mathbb{R}^{N}$, but here the role played by the dimension $N$ may be important (see [2]).

\section{Lyapunov inequality at higher eigenvalues}

If $n \in \mathbb{N}$ is fixed, we introduce the set

$$
\Lambda_{n}=\left\{a \in L^{1}(0, L): \lambda_{n} \prec a \text { and } 1.1 \text { has nontrivial solutions }\right\}
$$

Here $u \in H^{1}(0, L)$, the usual Sobolev space. If we define

$$
\beta_{1, n} \equiv \inf _{a \in \Lambda_{n}}\left\|a-\lambda_{n}\right\|_{L^{1}(0, L)},
$$

the main result of this section is the following.

\section{Theorem 2.1.}

$$
\beta_{1, n}=\frac{2 \pi n(n+1)}{L} \cot \frac{\pi n}{2(n+1)} .
$$

Moreover, $\beta_{1, n}$ is not attained.

Proof. It is based on some lemmas. In the first one we perform a careful analysis of the number and distribution of zeros of nontrivial solutions $u$ of (1.1). Since $a \in \Lambda_{n}$, it is clear that between two consecutive zeros of $u$ there must exist a zero of $u^{\prime}$, and between two consecutive zeros of $u^{\prime}$ there must exist a zero of $u$. More precisely, we have the following lemma.

Lemma 2.2. Let $a \in \Lambda_{n}$ be given and $u$ any nontrivial solution of 1 1.1. If the zeros of $u^{\prime}$ in $[0, L]$ are denoted by $0=x_{0}<x_{2}<\cdots<x_{2 m}=L$ and the zeros of $u$ in $(0, L)$ are denoted by $x_{1}<x_{3}<\cdots<x_{2 m-1}$, then:

(1) $x_{i+1}-x_{i} \leq L / 2 n$ for all $0 \leq i \leq 2 m-1$. Moreover, at least one of these inequalities is strict.

(2) $m \geq n+1$. Moreover, any value $m \geq n+1$ is possible. 
(3) Let $i, 0 \leq i \leq 2 m-1$, be given. Then the functions $a$ and $u$ satisfy

$$
\begin{aligned}
& \left\|a-\lambda_{n}\right\|_{L^{1}\left(x_{i}, x_{i+1}\right)} \geq \frac{\int_{x_{i}}^{x_{i+1}} u^{\prime 2}-\lambda_{n} \int_{x_{i}}^{x_{i+1}} u^{2}}{u^{2}\left(x_{i+1}\right)} \quad \text { if } i \text { is odd, } \\
& \left\|a-\lambda_{n}\right\|_{L^{1}\left(x_{i}, x_{i+1}\right)} \geq \frac{\int_{x_{i}}^{x_{i+1}} u^{\prime 2}-\lambda_{n} \int_{x_{i}}^{x_{i+1}} u^{2}}{u^{2}\left(x_{i}\right)} \quad \text { if } i \text { is even. }
\end{aligned}
$$

Proof. Let $i, 0 \leq i \leq 2 m-1$, be given. Then $u$ satisfies either the problem

$$
u^{\prime \prime}(x)+a(x) u(x)=0, \quad x \in\left(x_{i}, x_{i+1}\right), \quad u\left(x_{i}\right)=0, u^{\prime}\left(x_{i+1}\right)=0,
$$

or the problem

$$
u^{\prime \prime}(x)+a(x) u(x)=0, \quad x \in\left(x_{i}, x_{i+1}\right), \quad u^{\prime}\left(x_{i}\right)=0, u\left(x_{i+1}\right)=0 .
$$

Let us assume the first case. The reasoning in the second case is similar. Note that $u$ may be chosen such that $u(x)>0$ for all $x \in\left(x_{i}, x_{i+1}\right)$. Let us denote by $\mu_{1}^{i}$ and $\varphi_{1}^{i}$, respectively, the principal eigenvalue and eigenfunction of the eigenvalue problem

$$
v^{\prime \prime}(x)+\mu v(x)=0, \quad x \in\left(x_{i}, x_{i+1}\right), \quad v\left(x_{i}\right)=0, v^{\prime}\left(x_{i+1}\right)=0 .
$$

It is known that

$$
\mu_{1}^{i}=\frac{\pi^{2}}{4\left(x_{i+1}-x_{i}\right)^{2}}, \quad \varphi_{1}^{i}(x)=\sin \frac{\pi\left(x-x_{i}\right)}{2\left(x_{i+1}-x_{i}\right)} .
$$

Choosing $\varphi_{1}^{i}$ as test function in the weak formulation of 2.5 and $u$ as test function in the weak formulation of 2.7 for $\mu=\mu_{1}^{i}$ and $v=\varphi_{1}^{i}$, we obtain

$$
\int_{x_{i}}^{x_{i+1}}\left(a(x)-\mu_{1}^{i}\right) u \varphi_{1}^{i}(x) d x=0 .
$$

Then, if $x_{i+1}-x_{i}>L / 2 n$, we have

$$
\mu_{1}^{i}=\frac{\pi^{2} L^{2}}{4\left(x_{i+1}-x_{i}\right)^{2} L^{2}}<\frac{n^{2} \pi^{2}}{L^{2}}=\lambda_{n} \leq a(x) \quad \text { a.e. in }\left(x_{i}, x_{i+1}\right),
$$

which is a contradiction with (2.9). Consequently, $x_{i+1}-x_{i} \leq L / 2 n$ for all $0 \leq i \leq$ $2 m-1$. Also, since $\lambda_{n} \prec a$ in $(0, L)$, we must have $\lambda_{n} \prec a$ in some subinterval $\left(x_{j}, x_{j+1}\right)$. If $x_{j+1}-x_{j}=L / 2 n$, it follows that $\mu_{1}^{j} \prec a$ in $\left(x_{j}, x_{j+1}\right)$, and this again contradicts 2.9. This completes the proof of the first part of the lemma. For the second part, let us observe that

$$
L=\sum_{i=0}^{2 m-1}\left(x_{i+1}-x_{i}\right)<2 m \frac{L}{2 n}
$$

Consequently, $m>n$. Also, note that for any given natural number $q \geq n+1$, the function $a(x) \equiv \lambda_{q}$ belongs to $\Lambda_{n}$ and for $u(x)=\cos (q \pi x / L)$, we have $m=q$. 
Lastly, if $i$ with $0 \leq i \leq 2 m-1$ is given and $u$ satisfies (2.5), then

$$
\int_{x_{i}}^{x_{i+1}} u^{\prime 2}(x)=\int_{x_{i}}^{x_{i+1}} a(x) u^{2}(x)=\int_{x_{i}}^{x_{i+1}}\left(a(x)-\lambda_{n}\right) u^{2}(x)+\int_{x_{i}}^{x_{i+1}} \lambda_{n} u^{2}(x) .
$$

Therefore,

$$
\int_{x_{i}}^{x_{i+1}} u^{\prime 2}(x)-\lambda_{n} \int_{x_{i}}^{x_{i+1}} u^{2}(x) \leq\left\|a-\lambda_{n}\right\|_{L^{1}\left(x_{i}, x_{i+1}\right)}\left\|u^{2}\right\|_{L^{\infty}\left(x_{i}, x_{i+1}\right)}
$$

Since $u^{\prime}$ has no zeros in $\left(x_{i}, x_{i+1}\right)$ and $u\left(x_{i}\right)=0$, we have $\left\|u^{2}\right\|_{L^{\infty}\left(x_{i}, x_{i+1}\right)}=u^{2}\left(x_{i+1}\right)$. This proves the third part of the lemma when $u$ satisfies 2.5). The reasoning is similar if $u$ satisfies 2.6.

Lemma 2.3. Assume that $a<b$ and $0<M \leq \pi^{2} / 4(b-a)^{2}$ are given real numbers. Let $H=\left\{u \in H^{1}(a, b): u(a)=0, u(b) \neq 0\right\}$. If $J: H \rightarrow \mathbb{R}$ is defined by

$$
J(u)=\frac{\int_{a}^{b} u^{\prime 2}-M \int_{a}^{b} u^{2}}{u^{2}(b)}
$$

and $m \equiv \inf _{u \in H} J(u)$, then $m$ is attained. Moreover,

$$
m=M^{1 / 2} \cot \left(M^{1 / 2}(b-a)\right)
$$

and if $u \in H$, then $J(u)=m \Leftrightarrow u(x)=k \sin \left(M^{1 / 2}(x-a)\right) / \sin \left(M^{1 / 2}(b-a)\right)$ for some nonzero constant $k$.

Proof. Remember that $\delta_{1}=\pi^{2} / 4(b-a)^{2}$ is the principal eigenvalue of the eigenvalue problem $v^{\prime \prime}(x)+\delta v(x)=0, v(a)=0, v^{\prime}(b)=0$, with associated eigenfunction $w(x)=\sin \frac{\pi(x-a)}{2(b-a)}$. Therefore, if $M=\pi^{2} / 4(b-a)^{2}$, then $m=0$ and it is attained at the function $w$.

If $M<\delta_{1}=\pi^{2} / 4(b-a)^{2}$, there exists some positive constant $c$ such that

$$
\int_{a}^{b} u^{\prime 2}-M \int_{a}^{b} u^{2} \geq c \int_{a}^{b} u^{\prime 2}, \quad \forall u \in H
$$

If $\left\{u_{n}\right\} \subset H$ is a minimizing sequence for $J$, since the sequence $\left\{k_{n} u_{n}\right\}, k_{n} \neq 0$, is also a minimizing sequence, we can assume without loss of generality that $u_{n}(b)=1$. From 2.12 we deduce that $\int_{a}^{b} u_{n}^{\prime 2}$ is bounded. So, we can suppose that, up to a subsequence, $u_{n} \rightarrow u_{0}$ in $H^{1}(a, b)$ and $u_{n} \rightarrow u_{0}$ in $C[a, b]$ (with the uniform norm). The strong convergence in $C[a, b]$ gives us $u_{0}(b)=1$. The weak convergence in $H$ implies $J\left(u_{0}\right) \leq$ $\liminf J\left(u_{n}\right)=m$. Thus $u_{0}$ is a minimizer.

Since $J\left(u_{0}\right)=\min \left\{J(v): v \in H^{1}(a, b), v(a)=0, v(b)=1\right\}$, the Lagrange multiplier theorem implies that there are real numbers $\alpha_{1}, \alpha_{2}$ such that

$$
2 \int_{a}^{b} u_{0}^{\prime} v^{\prime}-2 M \int_{a}^{b} u_{0} v-\alpha_{1} v(b)-\alpha_{2} v(a)=0, \quad \forall v \in H^{1}(a, b) .
$$


In particular,

$$
\int_{a}^{b} u_{0}^{\prime} v^{\prime}-M \int_{a}^{b} u_{0} v=0, \quad \forall v \in H^{1}(a, b): v(a)=v(b)=0 .
$$

We conclude that $u_{0}$ satisfies the problem

$$
u_{0}^{\prime \prime}(x)+M u_{0}(x)=0, \quad x \in(a, b), \quad u_{0}(a)=0, u_{0}(b)=1 .
$$

Note that since $M<\pi^{2} /(b-a)^{2}, 2.13$ has a unique solution, which is given by

$$
u_{0}(x)=\frac{\sin \left(M^{1 / 2}(x-a)\right)}{\sin \left(M^{1 / 2}(b-a)\right)} .
$$

Finally, an elementary calculation gives $J\left(u_{0}\right)=M^{1 / 2} \cot \left(M^{1 / 2}(b-a)\right)$. This proves the lemma.

Now, we combine Lemmas 2.2 and 2.3 to obtain the following result.

Lemma 2.4. Let $a \in \Lambda_{n}$ be given and $u$ any nontrivial solution of (1.1). If the zeros of $u^{\prime}$ are denoted by $0=x_{0}<x_{2}<\cdots<x_{2 m}=L$ and the zeros of $u$ are denoted by $x_{1}<x_{3}<\cdots<x_{2 m-1}$, then

$$
\left\|a-\lambda_{n}\right\|_{L^{1}(0, L)} \geq \frac{n \pi}{L} \sum_{i=0}^{2 m-1} \cot \left(\frac{n \pi}{L}\left(x_{i+1}-x_{i}\right)\right) .
$$

The previous reasoning motivates the study of a special minimization problem given in the following lemma.

Lemma 2.5. Given any $r \in \mathbb{N}$ and $S \in \mathbb{R}^{+}$satisfying $r \pi>2 S$, let

$$
Z=\left\{z=\left(z_{0}, z_{1}, \ldots, z_{r-1}\right) \in(0, \pi / 2]^{r}: \sum_{i=0}^{r-1} z_{i}=S\right\} .
$$

If $F: Z \rightarrow \mathbb{R}$ is defined by

$$
F(z)=\sum_{i=0}^{r-1} \cot z_{i}
$$

then $\inf _{z \in Z} F(z)$ is attained and its value is $r \cot (S / r)$. Moreover, $z \in Z$ is a minimizer if and only if $z_{i}=S / r$ for all $0 \leq i \leq r-1$.

Proof. Let us observe that for all $z \in Z$, $\cot z_{i} \geq 0$ for $0 \leq i \leq r-1$. Moreover, if $z_{i} \rightarrow 0^{+}$for some $0 \leq i \leq r-1$, then $\cot z_{i} \rightarrow+\infty$. Also, since $r \pi>2 S$, if $z \in Z$ is such that $z_{i}=\pi / 2$ for some $0 \leq i \leq r-1$, then there must exist some $0 \leq j \leq r-1$ such that $z_{j}<\pi / 2$. Let us choose the point $z^{*} \in Z$ defined (for $\delta>0$ sufficiently small) by $z_{k}^{*}=z_{k}$, if $k \neq i$ and $k \neq j, z_{i}^{*}=\pi / 2-\delta, z_{j}^{*}=z_{j}+\delta$. An elementary calculation shows

$$
F\left(z^{*}\right)-F(z)=\frac{\cot z_{j}\left(1-\cot z_{j} \cot \delta\right)}{\cot \delta\left(\cot z_{j}+\cot \delta\right)},
$$


which is a negative number for $\delta$ sufficiently small. Consequently, there exists a sufficiently small positive constant $\varepsilon_{1}$ such that

$$
\inf _{z \in Z} F(z)=\min _{z \in\left[\varepsilon_{1}, \pi / 2\right]^{r}} F(z)=\min _{z \in\left(\varepsilon_{1}, \pi / 2\right)^{r}} F(z) .
$$

Then, if $z \in Z$ is any minimizer of $F$, the Lagrange multiplier theorem implies that there is $\lambda \in \mathbb{R}$ such that

$$
\frac{-1}{\sin ^{2} z_{i}}+\lambda=0, \quad 0 \leq i \leq r-1, \quad \sum_{i=0}^{r-1} z_{i}=S .
$$

We conclude that $z_{i}=S / r, 0 \leq i \leq r-1$, and the lemma is proved.

From the previous two lemmas, we obtain the following one.

Lemma 2.6.

$$
\beta_{1, n} \geq \frac{n \pi}{L} 2(n+1) \cot \frac{n \pi}{2(n+1)} .
$$

Proof. Let $a \in \Lambda_{n}$ be given and $u$ any nontrivial solution of 1.1p. If the zeros of $u^{\prime}$ are denoted by $0=x_{0}<x_{2}<\cdots<x_{2 m}=L$ and the zeros of $u$ are denoted by $x_{1}<x_{3}<\cdots<x_{2 m-1}$, then from Lemmas 2.4 and 2.5 (with $r=2 m, S=n \pi$ and $\left.z_{i}=\frac{n \pi}{L}\left(x_{i+1}-x_{i}\right)\right)$ we obtain

$$
\left\|a-\lambda_{n}\right\|_{L^{1}(0, L)} \geq \frac{n \pi}{L} \sum_{i=0}^{2 m-1} \cot \left(\frac{n \pi}{L}\left(x_{i+1}-x_{i}\right)\right) \geq \frac{n \pi}{L} 2 m \cot \frac{n \pi}{2 m} .
$$

Finally, taking into account that

$$
\text { the function } 2 m \cot \frac{n \pi}{2 m} \text { is strictly increasing with respect to } m
$$

and that $m \geq n+1$, we deduce 2.16.

In the next lemma, we define a minimizing sequence for $\beta_{1, n}$.

Lemma 2.7. Let $\varepsilon>0$ be sufficiently small. Define $u_{\varepsilon}:[0, L] \rightarrow \mathbb{R}$ by

$$
u_{\varepsilon}(x)= \begin{cases}-\sin \left(\frac{n \pi}{L}\left(x-\frac{L}{2(n+1)}\right)\right) & +\frac{n \pi}{L} \frac{(x-\varepsilon)^{3}}{3 \varepsilon^{2}} \cos \left(\frac{n \pi}{2(n+1)}\right) \text { if } 0 \leq x \leq \varepsilon, \\ -\sin \left(\frac{n \pi}{L}\left(x-\frac{L}{2(n+1)}\right)\right) & \text { if } \varepsilon \leq x \leq \frac{L}{2(n+1)}, \\ -u_{\varepsilon}\left(\frac{2 L}{2(n+1)}-x\right) & \text { if } \frac{L}{2(n+1)} \leq x \leq \frac{2 L}{2(n+1)}, \\ u_{\varepsilon}\left(\frac{4 L}{2(n+1)}-x\right) & \text { if } \frac{2 L}{2(n+1)} \leq x \leq \frac{4 L}{2(n+1)}, \\ -u_{\varepsilon}\left(\frac{6 L}{2(n+1)}-x\right) & \text { if } \frac{4 L}{2(n+1)} \leq x \leq \frac{6 L}{2(n+1)}, \\ \ldots & \end{cases}
$$


Then $u_{\varepsilon} \in C^{2}[0, L]$, the function $a_{\varepsilon}(x) \equiv-u_{\varepsilon}^{\prime \prime}(x) / u_{\varepsilon}(x)$ for all $x \in[0, L], x \neq \frac{(2 k-1) L}{2(n+1)}$, $1 \leq k \leq n+1$, belongs to $\Lambda_{n}$ and

$$
\liminf _{\varepsilon \rightarrow 0^{+}}\left\|a_{\varepsilon}-\lambda_{n}\right\|_{L^{1}(0, L)}=\frac{n \pi}{L} 2(n+1) \cot \frac{n \pi}{2(n+1)} .
$$

Proof. We claim that for each $0 \leq i \leq 2 n+1$, the function $a_{\varepsilon}$ satisfies

$$
\lambda_{n} \prec a_{\varepsilon} \quad \text { in }\left(\frac{i L}{2(n+1)}, \frac{(i+1) L}{2(n+1)}\right),
$$

and

$$
\liminf _{\varepsilon \rightarrow 0^{+}}\left\|a_{\varepsilon}-\lambda_{n}\right\|_{L^{1}\left(\frac{i L}{2(n+1)}, \frac{(i+1) L}{2(n+1)}\right)}=\frac{n \pi}{L} \cot \frac{n \pi}{2(n+1)} .
$$

It is trivial that 2.19 follows from 2.20 ) and 2.21). Moreover, taking into account the definition of $u_{\varepsilon}$, it is clear that it is sufficient to prove the claim in the case $i=0$. Now, if $x \in(0, L / 2(n+1))$ we can distinguish two cases:

(1) $x \in(\varepsilon, L / 2(n+1))$. Then $a_{\varepsilon}(x)=-u_{\varepsilon}^{\prime \prime}(x) / u_{\varepsilon}(x) \equiv \lambda_{n}$.

(2) $x \in(0, \varepsilon)$. Then

$$
a_{\varepsilon}(x)-\lambda_{n}=\frac{-2 \frac{x-\varepsilon}{\varepsilon^{2}} \frac{n \pi}{L} \cos \frac{n \pi}{2(n+1)}-\frac{(x-\varepsilon)^{3}}{3 \varepsilon^{2}} \frac{n^{3} \pi^{3}}{L^{3}} \cos \frac{n \pi}{2(n+1)}}{-\sin \left(\frac{n \pi}{L}\left(x-\frac{L}{2(n+1)}\right)\right)+\frac{(x-\varepsilon)^{3}}{3 \varepsilon^{2}} \frac{n \pi}{L} \cos \frac{n \pi}{2(n+1)}}>0 .
$$

Therefore $a_{\varepsilon} \in \Lambda_{n}$. Moreover, if $\varepsilon \rightarrow 0^{+}$, then

$$
\frac{-\frac{(x-\varepsilon)^{3}}{3 \varepsilon^{2}} \frac{n^{3} \pi^{3}}{L^{3}} \cos \frac{n \pi}{2(n+1)}}{-\sin \left(\frac{n \pi}{L}\left(x-\frac{L}{2(n+1)}\right)\right)+\frac{(x-\varepsilon)^{3}}{3 \varepsilon^{2}} \frac{n \pi}{L} \cos \frac{n \pi}{2(n+1)}} \rightarrow 0,
$$

uniformly in $x \in(0, \varepsilon)$.

Finally, since

$\lim _{\varepsilon \rightarrow 0^{+}} \int_{0}^{\varepsilon}\left[\frac{-2 \frac{x-\varepsilon}{\varepsilon^{2}} \frac{n \pi}{L} \cos \frac{n \pi}{2(n+1)}}{-\sin \left(\frac{n \pi}{L}\left(x-\frac{L}{2(n+1)}\right)\right)+\frac{(x-\varepsilon)^{3}}{3 \varepsilon^{2}} \frac{n \pi}{L} \cos \frac{n \pi}{2(n+1)}}-\frac{-2 \frac{x-\varepsilon}{\varepsilon^{2}} \frac{n \pi}{L} \cos \frac{n \pi}{2(n+1)}}{-\sin \left(\frac{n \pi}{L}\left(x-\frac{L}{2(n+1)}\right)\right)}\right]=0$,

and

$$
-\sin \left(\frac{n \pi}{L}\left(x-\frac{L}{2(n+1)}\right)\right) \rightarrow \sin \frac{n \pi}{2(n+1)}
$$

uniformly in $x \in(0, \varepsilon)$ when $\varepsilon \rightarrow 0+$, we deduce

$$
\begin{aligned}
\liminf _{\varepsilon \rightarrow 0^{+}}\left\|a_{\varepsilon}-\lambda_{n}\right\|_{L^{1}\left(0, \frac{L}{2(n+1)}\right)} & =\liminf _{\varepsilon \rightarrow 0^{+}} \frac{n \pi}{L} \cot \frac{n \pi}{2(n+1)} \frac{2}{\varepsilon^{2}} \int_{0}^{\varepsilon}(\varepsilon-x) d x \\
& =\frac{n \pi}{L} \cot \frac{n \pi}{2(n+1)},
\end{aligned}
$$

which is 2.21) for the case $i=0$. 
Lemma 2.8. $\beta_{1, n}$ is not attained.

Proof. Let $a \in \Lambda_{n}$ be such that $\left\|a-\lambda_{n}\right\|_{L^{1}(0, L)}=\beta_{1, n}$. Let $u$ be any nontrivial solution of (1.1) associated to the function $a$. As previously, we denote the zeros of $u^{\prime}$ by $0=x_{0}<$ $x_{2}<\cdots<x_{2 m}=L$ and the zeros of $u$ by $x_{1}<x_{3}<\cdots<x_{2 m-1}$. By using Lemmas 2.4, 2.5 and 2.7, we have

$$
\begin{aligned}
\beta_{1, n} & =\left\|a-\lambda_{n}\right\|_{L^{1}(0, L)}=\sum_{i=0}^{2 m-1}\left\|a-\lambda_{n}\right\|_{L^{1}\left(x_{i}, x_{i+1}\right)} \\
& \geq \sum_{i=0}^{2 m-1} J_{i}(u) \geq \frac{n \pi}{L} \sum_{i=0}^{2 m-1} \cot \frac{n \pi\left(x_{i+1}-x_{i}\right)}{L} \\
& \geq \frac{n \pi}{L} 2 m \cot \frac{n \pi}{2 m} \geq \frac{n \pi}{L} 2(n+1) \cot \frac{n \pi}{2(n+1)}=\beta_{1, n}
\end{aligned}
$$

where $J_{i}(u)$ is given either by

$$
J_{i}(u)=\frac{\int_{x_{i}}^{x_{i+1}} u^{\prime 2}-\lambda_{n} \int_{x_{i}}^{x_{i+1}} u^{2}}{u^{2}\left(x_{i+1}\right)} \quad \text { if } u\left(x_{i}\right)=0
$$

or by

$$
J_{i}(u)=\frac{\int_{x_{i}}^{x_{i+1}} u^{\prime 2}-\lambda_{n} \int_{x_{i}}^{x_{i+1}} u^{2}}{u^{2}\left(x_{i}\right)} \quad \text { if } u\left(x_{i+1}\right)=0 .
$$

Consequently, all inequalities in 2.22) transform into equalities. In particular, from Lemma 2.5 and the property $(\mathrm{P})$ shown in Lemma 2.6 we obtain

$$
m=n+1, \quad x_{i+1}-x_{i}=\frac{L}{2(n+1)}, \quad 0 \leq i \leq 2 n+1 .
$$

Also, it follows that

$$
J_{i}(u)=\frac{n \pi}{L} \cot \frac{n \pi}{L} \frac{L}{2(n+1)}, \quad 0 \leq i \leq 2 n+1 .
$$

From Lemma 2.3 we deduce that, up to some nonzero constants, in each interval $\left[x_{i}, x_{i+1}\right]$,

$$
u(x)= \begin{cases}\frac{\sin \frac{n \pi}{L}\left(x-x_{i}\right)}{\sin \frac{n \pi}{L}\left(x_{i+1}-x_{i}\right)} & \text { if } i \text { is odd, } \\ \frac{\sin \frac{n \pi}{L}\left(x-x_{i+1}\right)}{\sin \frac{n \pi}{L}\left(x_{i}-x_{i+1}\right)} & \text { if } i \text { is even. }\end{cases}
$$

In particular, in $[0, L / 2(n+1)], u$ must be the function

$$
u(x)=\frac{\sin \frac{n \pi}{L}\left(x-\frac{L}{2(n+1)}\right)}{\sin \frac{n \pi}{L}\left(-\frac{L}{2(n+1)}\right)},
$$

which does not satisfy the condition $u^{\prime}(0)=0$. The conclusion is that $\beta_{1, n}$ is not attained. 
Finally, as a trivial consequence of Lemmas 2.6, 2.7 and 2.8 we have the conclusion of Theorem 2.1 .

Remark 1. Let us observe that if we consider $\beta_{1, n}$ as a function of $n \in(0,+\infty)$, then $\lim _{n \rightarrow 0^{+}} \beta_{1, n}=4 / L$, the constant of the classical $L^{1}$ Lyapunov inequality at the first eigenvalue ([5]).

Remark 2. The case where $L=1$ and the function $a$ satisfies the condition $A \leq a(x) \leq$ $B$, a.e. in $(0, L)$ where $\lambda_{k}<A<\lambda_{k+1} \leq B$ for some $k \in \mathbb{N} \cup\{0\}$, has been considered in [6], where the authors use optimal control theory methods. They define $\Lambda_{A, B}$ to be the set of $a \in L^{1}(0, L)$ such that $A \leq a(x) \leq B$ a.e. in $(0, L)$ and 1.1 has nontrivial solutions. Then, by using Pontryagin's maximum principle, they prove that the number

$$
\beta_{A, B} \equiv \inf _{a \in \Lambda_{A, B}}\|a\|_{L^{1}(0, L)}
$$

is attained. In addition, they calculate $\lim _{B \rightarrow+\infty} \beta_{A, B}$.

Remark 3. In our opinion, the inequality $\int_{0}^{1} b(t) d t \leq 2 \sqrt{A} \cot (\sqrt{A} / 2)$ in 6 , Theorem 3] must be substituted by $\int_{0}^{1} b(t) d t \leq A+2(k+1) \sqrt{A} \cot \frac{\sqrt{A}}{2(k+1)}$. This may be easily derived from our method by modifying the definition of the set $\Lambda_{n}$ (given in 2.1 ) in a trivial way.

Remark 4. If $A \rightarrow \lambda_{k}{ }^{+}$, it does not seem possible to deduce from [6] that the constant $\beta_{1, k}$ (defined in (2.2) ) is not attained. In fact, to the best of our knowledge, this result is new. Moreover, our method, which combines a detailed analysis of the number and distribution of zeros of nontrivial solutions of (1.1) and their first derivatives with the use of suitable minimization problems, will be very useful to combine Lyapunov inequalities and disfocality. This will be seen in the next section.

Remark 5. We can use our methods to make an analogous study for other boundary conditions. In particular, with the help of Lemmas 2.2 and 2.3 we can consider the mixed linear problem

$$
u^{\prime \prime}(x)+a(x) u(x)=0, \quad x \in(0, L), \quad u^{\prime}(0)=u(L)=0
$$

where

$$
a \in \Gamma_{n}=\left\{a \in L^{1}(0, L): \mu_{n} \prec a \text { and } 2.23 \text { has nontrivial solutions }\right\} .
$$

Here $\mu_{n}$ is the $n$-th eigenvalue of the eigenvalue problem

$$
u^{\prime \prime}(x)+\mu u(x)=0, \quad x \in(0, L), \quad u^{\prime}(0)=u(L)=0 .
$$

The case where $L=1$ and the function $a$ satisfies the condition $A \leq a(x) \leq B$, a.e. in $(0, L)$ where $\mu_{k}<A<\mu_{k+1} \leq B$ has been considered in [7]. As in [6], the authors use optimal control theory methods. See also [12] for Dirichlet boundary conditions. 


\section{Lyapunov inequalities and disfocality}

The $L^{\infty}$ Lyapunov inequality is trivial from Dolph's result ([4] $)$. In fact, by using Dolph's result, the constant

$$
\beta_{\infty, n} \equiv \inf _{a \in \Lambda_{n}}\|a\|_{L^{\infty}(0, L)}
$$

must be greater than or equal to $\lambda_{n+1}$. Since the constant function $\lambda_{n+1}$ is an element of $\Lambda_{n}$, we deduce

$$
\beta_{\infty, n}=\lambda_{n+1} .
$$

Moreover, $\beta_{\infty, n}$ is attained at a unique element $a_{\infty} \in \Lambda_{n}$ given by the constant function $a_{\infty} \equiv \lambda_{n+1}$. On the other hand, under the restriction

$$
a \in L^{1}(0, L), \quad \lambda_{n} \prec a,
$$

the relation between Neumann boundary conditions and disfocality arises in a natural way. In fact, if $u \in H^{1}(0, L)$ is any nontrivial solution of 1.1 and the zeros of $u$ are denoted by $x_{1}<x_{3}<\cdots<x_{2 m-1}$, and the zeros of $u^{\prime}$ are denoted by $0=x_{0}<x_{2}<$ $\cdots<x_{2 m}=L$, then for each $i, 0 \leq i \leq 2 m-1$, the function $u$ satisfies

$$
u^{\prime \prime}(x)+a(x) u(x)=0, x \in\left(x_{i}, x_{i+1}\right), \quad u\left(x_{i}\right)=0, u^{\prime}\left(x_{i+1}\right)=0, \quad \text { if } i \text { is odd }
$$

and

$$
u^{\prime \prime}(x)+a(x) u(x)=0, x \in\left(x_{i}, x_{i+1}\right), \quad u^{\prime}\left(x_{i}\right)=0, u\left(x_{i+1}\right)=0, \quad \text { if } i \text { is even. }
$$

Consequently, each of the problems (3.4) and 3.5 with $0 \leq i \leq 2 m-1$ has a nontrivial solution. This simple observation can be used to deduce the following conclusion: if $a$ is any function satisfying (3.3) such that for any $m \geq n+1$ and any distribution of numbers $0=x_{0}<x_{1}<x_{2}<\cdots<x_{2 m-1}<x_{2 m}=L$, either some problem of the type (3.4) or some problem of the type (3.5) has only the trivial solution, then problem (1.1) has only the trivial solution. Lastly, it has been established in [3] (Theorem 2.1 for the case $p=\infty)$ that if $b \in L^{\infty}(c, d)$ satisfies

$$
\|b\|_{L^{\infty}(c, d)} \leq \frac{\pi^{2}}{4(d-c)^{2}} \quad \text { and } \quad b \neq \frac{\pi^{2}}{4(d-c)^{2}} \quad \text { in }(c, d),
$$

then the unique solution of the boundary value problems

$$
u^{\prime \prime}(x)+b(x) u(x)=0, \quad x \in(c, d), \quad u^{\prime}(c)=u(d)=0,
$$

and

$$
u^{\prime \prime}(x)+b(x) u(x)=0, \quad x \in(c, d), \quad u(c)=u^{\prime}(d)=0
$$

is the trivial one.

We may use the previous reasonings to obtain the following result: 
Theorem 3.1. If $a \in L^{\infty}(0, L), \lambda_{n} \prec a$ and there exists $0=y_{0}<y_{1}<\cdots<y_{2 n+1}<$ $y_{2 n+2}=L$ such that

$$
\max _{0 \leq i \leq 2 n+1}\left\{\left(y_{i+1}-y_{i}\right)^{2}\|a\|_{L^{\infty}\left(y_{i}, y_{i+1}\right)}\right\} \leq \pi^{2} / 4,
$$

and in addition $a$ is not the constant $\pi^{2} / 4\left(y_{i+1}-y_{i}\right)^{2}$ in at least one of the intervals $\left[y_{i}, y_{i+1}\right], 0 \leq i \leq 2 n+1$, then the boundary value problem (1.1) has only the trivial solution.

Proof. If $m \geq n+1$ and $0=x_{0}<x_{1}<x_{2}<\cdots<x_{2 m-1}<x_{2 m}=L$ is any distribution of numbers, then either

$$
\left[x_{j}, x_{j+1}\right] \subset\left[y_{i}, y_{i+1}\right], \quad \text { strictly, }
$$

for some $0 \leq i \leq 2 n+1,0 \leq j \leq 2 m-1$, or

$$
m=n+1 \quad \text { and } \quad x_{i}=y_{i}, \quad \forall 0 \leq i \leq 2 n+2 .
$$

If 3.10$]$ is satisfied, then

$$
\|a\|_{L^{\infty}\left(x_{j}, x_{j+1}\right)}<\|a\|_{L^{\infty}\left(y_{i}, y_{i+1}\right)} \leq \frac{\pi^{2}}{4\left(y_{i+1}-y_{i}\right)^{2}}<\frac{\pi^{2}}{4\left(x_{j+1}-x_{j}\right)^{2}}
$$

and consequently we deduce from $(3.4)-(3.6)$ that $(1.1)$ has only the trivial solution.

If $(3.11)$ is satisfied, we deduce from the hypotheses of the theorem that $a$ is not the constant $\pi^{2} / 4\left(x_{i+1}-x_{i}\right)^{2}$ in at least one of the intervals $\left[x_{i}, x_{i+1}\right], 0 \leq i \leq 2 n+1$. Therefore, again (3.4)-3.6 imply that (1.1) has only the trivial solution. In any case, we have the desired conclusion.

Remark 6. If in the previous theorem we choose $y_{i}=i L / 2(n+1), 0 \leq i \leq 2 n+2$, then we have the so called nonuniform nonresonance conditions at higher eigenvalues ([4], [10]) but if for instance, $y_{j+1}-y_{j}<L / 2(n+1)$ for some $j, 0 \leq j \leq 2 n+1$, the function $a$ can satisfy $\|a\|_{L^{\infty}\left(y_{j}, y_{j+1}\right)}=\pi^{2} / 4\left(y_{j+1}-y_{j}\right)^{2}$ (which is a quantity greater than $\left.\lambda_{n+1}=(n+1)^{2} \pi^{2} / L^{2}\right)$ as long as $a$ satisfies 3.9 for each $i \neq j$.

Remark 7. The hypothesis of the previous theorem is optimal in the sense that if $a$ is the constant $\pi^{2} / 4\left(y_{i+1}-y_{i}\right)^{2}$ in each of the intervals $\left(y_{i}, y_{i+1}\right), 0 \leq i \leq 2 n+1$, then (1.1) has nontrivial solutions. In fact, if this is the case, it is easily checked that there exist appropriate constants $k_{i}, 0 \leq i \leq 2 n+1$, such that the function

$$
u(x)= \begin{cases}k_{i} \cos \frac{\pi\left(x-y_{i}\right)}{2\left(y_{i+1}-y_{i}\right)}, & x \in\left[y_{i}, y_{i+1}\right], i \text { even, } \\ k_{i} \cos \frac{\pi\left(y_{i+1}-x\right)}{2\left(y_{i+1}-y_{i}\right)}, & x \in\left[y_{i}, y_{i+1}\right], i \text { odd },\end{cases}
$$

is a nontrivial solution of 1.1 . 
Now we comment on some relations between the Lyapunov constant $\beta_{1, n}$ given in Theorem 2.1 and disfocality. It is clear from the definition of $\beta_{1, n}$ that if a function $a$ satisfies

$$
a \in L^{1}(0, L), \quad \lambda_{n} \prec a, \quad\left\|a-\lambda_{n}\right\|_{1}<\beta_{1, n},
$$

then the unique solution of $(1.1)$ is the trivial one. In the next theorem we prove that, with the use of disfocality, we can obtain a more general condition.

\section{Theorem 3.2.}

(1) If $a \in L^{1}(0, L), \lambda_{n} \prec a$, and there exist $0=y_{0}<y_{1}<\cdots<y_{2 n+1}<y_{2 n+2}=L$ such that

$$
y_{i+1}-y_{i}<\frac{L}{2 n} ;\left\|a-\lambda_{n}\right\|_{L^{1}\left(y_{i}, y_{i+1}\right)}<\frac{n \pi}{L} \cot \frac{n \pi\left(y_{i+1}-y_{i}\right)}{L}, \forall 0 \leq i \leq 2 n+1,
$$

then the unique solution of (1.1) is the trivial one.

(2) 3.13 implies 3.14 .

(3) If $0=y_{0}<y_{1}<\cdots<y_{2 n+1}<y_{2 n+2}=L$ is any distribution of numbers such that $y_{k+1}-y_{k}<L / 2 n$ for all $0 \leq k \leq 2 n+1$ and $y_{i+1}-y_{i} \neq y_{j+1}-y_{j}$ for some $0 \leq i, j \leq 2 n+1$, then there exists $a \in L^{1}(0, L)$ with $\lambda_{n} \prec a$, satisfying 3.14 but not 3.13.

Proof. If $a$ satisfies (3.14), then the unique solution of (1.1) is the trivial one. In fact, if this is not true, let $u$ be a nontrivial solution of (1.1) and denote the zeros of $u$ by $x_{1}<x_{3}<\cdots<x_{2 m-1}$ and the zeros of $u^{\prime}$ by $0=x_{0}<x_{2}<\cdots<x_{2 m}=L$. Since $m \geq n+1$,

$$
\left[x_{j}, x_{j+1}\right] \subset\left[y_{i}, y_{i+1}\right]
$$

for some $0 \leq i \leq 2 n+1,0 \leq j \leq 2 m-1$. Consequently,

$$
\frac{\left\|a-\lambda_{n}\right\|_{L^{1}\left(x_{j}, x_{j+1}\right)}}{\cot \frac{n \pi\left(x_{j+1}-x_{j}\right)}{L}} \leq \frac{\left\|a-\lambda_{n}\right\|_{L^{1}\left(y_{i}, y_{i+1}\right)}}{\cot \frac{n \pi\left(y_{i+1}-y_{i}\right)}{L}}<\frac{n \pi}{L} .
$$

From this we deduce

$$
\left\|a-\lambda_{n}\right\|_{L^{1}\left(x_{j}, x_{j+1}\right)}<\frac{n \pi}{L} \cot \frac{n \pi\left(x_{j+1}-x_{j}\right)}{L},
$$

which is a contradiction with Lemmas 2.2 and 2.3 .

Next we prove that 3.13 implies 3.14 . We can certainly assume that inf $a>\lambda_{n}$, for if not, we replace $a$ by $a+\delta$ (for small $\delta>0$ ) and the new function $a+\delta$ satisfies (3.13). Note that if condition (3.14) is satisfied for $a+\delta$ then it is also satisfied for $a$.

Now choose $\varepsilon>0$ sufficiently small. Since the function

$$
\frac{\left\|a-\lambda_{n}\right\|_{L^{1}(0, y)}}{\cot \frac{n \pi(y-0)}{L}}
$$


is strictly increasing with respect to $y \in(0, L / 2 n)$ and

$$
\lim _{y \rightarrow 0^{+}} \frac{\left\|a-\lambda_{n}\right\|_{L^{1}(0, y)}}{\cot \frac{n \pi(y-0)}{L}}=0, \quad \lim _{y \rightarrow L^{-}} \frac{\left\|a-\lambda_{n}\right\|_{L^{1}(0, y)}}{\cot \frac{n \pi(y-0)}{L}}=+\infty,
$$

there is a unique $y_{1}, 0=y_{0}<y_{1}<L / 2 n$, such that

$$
\frac{\left\|a-\lambda_{n}\right\|_{L^{1}\left(0, y_{1}\right)}}{\cot \frac{n \pi\left(y_{1}-0\right)}{L}}=\frac{n \pi}{L}-\varepsilon
$$

With the help of a similar reasoning, it is possible to prove the existence of points $0=$ $y_{0}<y_{1}<\cdots<y_{2 n+1}$ such that

$$
\frac{\left\|a-\lambda_{n}\right\|_{L^{1}\left(y_{i}, y_{i+1}\right)}}{\cot \frac{n \pi\left(y_{i+1}-y_{i}\right)}{L}}=\frac{n \pi}{L}-\varepsilon, \quad y_{i+1}-y_{i}<\frac{L}{2 n}, \quad 0 \leq i \leq 2 n .
$$

(If necessary, we can define $a(x)=\lambda_{n}$ for all $x>L$ ).

Since $y_{i+1}-y_{i}<L / 2 n$ for all $0 \leq i \leq 2 n-1$, we have $y_{2 n}<L$.

If $y_{2 n+1} \geq L$, then we replace $y_{2 n+1}$ with $y_{2 n+1}=L-\mu$ (for small $\mu>0$ ). Finally, choosing $y_{2 n+2}=L$, we obtain (3.14).

If $y_{2 n+1}<L$, take $y_{2 n+2}=L$. We claim that

$$
y_{2 n+2}-y_{2 n+1}<\frac{L}{2 n} \quad \text { and } \quad \frac{\left\|a-\lambda_{n}\right\|_{L^{1}\left(y_{2 n+1}, y_{2 n+2}\right)}}{\cot \frac{n \pi\left(y_{2 n+2}-y_{2 n+1}\right)}{L}}<\frac{n \pi}{L}-\varepsilon .
$$

In fact, if $y_{2 n+2}-y_{2 n+1} \geq L / 2 n$, then $y_{2 n+1} \leq L(2 n-1) / 2 n$. Then, from (3.17), Lemma 2.5 (with $r=2 n+1, S=\frac{n \pi}{L}\left(y_{2 n+1}\right)$ and $z_{i}=\frac{n \pi}{L}\left(y_{i+1}-y_{i}\right)$ ) and using the monotonicity of $\cot$ in $(0, \pi / 2)$ we obtain

$$
\begin{aligned}
\frac{n \pi}{L} 2(n & +1) \cot \frac{n \pi}{2(n+1)}=\beta_{1, n}>\sum_{i=0}^{2 n}\left\|a-\lambda_{n}\right\|_{L^{1}\left(y_{i}, y_{i+1}\right)} \\
& =\left(\frac{n \pi}{L}-\varepsilon\right) \sum_{i=0}^{2 n} \cot \frac{n \pi}{L}\left(y_{i+1}-y_{i}\right) \geq\left(\frac{n \pi}{L}-\varepsilon\right)(2 n+1) \cot \frac{n \pi}{L(2 n+1)} y_{2 n+1} \\
& \geq\left(\frac{n \pi}{L}-\varepsilon\right)(2 n+1) \cot \frac{\pi(2 n-1)}{2(2 n+1)} .
\end{aligned}
$$

If $\varepsilon \rightarrow 0^{+}$, we conclude

$$
\beta_{1, n} \geq \frac{n \pi}{L}(2 n+1) \cot \frac{\pi(2 n-1)}{2(2 n+1)} .
$$

Now, since the function $x \mapsto \frac{2 \pi \cot x}{\pi-2 x}$ is strictly decreasing in $(0, \pi / 2)$ and $\frac{\pi(2 n-1)}{2(2 n+1)}<$ $\frac{n \pi}{2(n+1)}$, we obtain

$$
\beta_{1, n} \geq \frac{n \pi}{L}(2 n+1) \cot \frac{\pi(2 n-1)}{2(2 n+1)}>\frac{n \pi}{L} 2(n+1) \cot \frac{n \pi}{2(n+1)}=\beta_{1, n},
$$

which is a contradiction. 
It remains to prove the second part of the claim (3.18). In fact, if it is not true, then from 3.17 and Lemma 2.5 (with $r=2 n+2, S=n \pi$ and $z_{i}=\frac{n \pi}{L}\left(y_{i+1}-y_{i}\right)$ ) we have

$$
\begin{aligned}
\left\|a-\lambda_{n}\right\|_{L^{1}(0, L)} & =\sum_{i=0}^{2 n+1}\left\|a-\lambda_{n}\right\|_{L^{1}\left(y_{i}, y_{i+1}\right)} \\
& \geq\left(\frac{n \pi}{L}-\varepsilon\right) \sum_{i=0}^{2 n+1} \cot \frac{n \pi\left(y_{i+1}-y_{i}\right)}{L} \geq\left(\frac{n \pi}{L}-\varepsilon\right) \frac{\beta_{1, n}}{n \pi / L}
\end{aligned}
$$

for $\varepsilon>0$ sufficiently small. This is a contradiction with 3.13 .

Finally, to prove (3), take $0=y_{0}<y_{1}<\cdots<y_{2 n+1}<y_{2 n+2}=L$ such that $y_{k+1}-y_{k}<L / 2 n$ for all $0 \leq k \leq 2 n+1$ and $y_{i+1}-y_{i} \neq y_{j+1}-y_{j}$ for some $0 \leq i, j \leq 2 n+1$. Then from Lemma 2.5 we obtain

$$
\sum_{i=0}^{2 n+1} \frac{n \pi}{L} \cot \frac{n \pi\left(y_{i+1}-y_{i}\right)}{L}>\frac{2 \pi n(n+1)}{L} \cot \frac{n \pi}{2(n+1)}=\beta_{1, n} .
$$

Now, choose $a \in L^{1}(0, L)$ with $\lambda_{n} \prec a$ satisfying

$$
\left\|a-\lambda_{n}\right\|_{L^{1}\left(y_{i}, y_{i+1}\right)}=\frac{n \pi}{L} \cot \frac{n \pi\left(y_{i+1}-y_{i}\right)}{L}-\varepsilon, \quad \forall 0 \leq i \leq 2 n+1 .
$$

It is trivial that if $\varepsilon$ is sufficiently small, then $a$ satisfies 3.14 whereas

$$
\left\|a-\lambda_{n}\right\|_{L^{1}(0, L)}=\sum_{i=0}^{2 n+1}\left\|a-\lambda_{n}\right\|_{L^{1}\left(y_{i}, y_{i+1}\right)}>\beta_{1, n}
$$

\section{Final remark on nonlinear problems}

We finish this paper by showing how to use previous reasonings to obtain new theorems on the existence and uniqueness of solutions of nonlinear b.v.p.

$$
u^{\prime \prime}(x)+f(x, u(x))=0, \quad x \in(0, L), \quad u^{\prime}(0)=u^{\prime}(L)=0 .
$$

For example, we have the following theorem related to Theorem 2.1 in [10]. This last theorem allows one to consider more general boundary value problems, but for ordinary problems with Neumann boundary conditions our hypotheses allow a more general behavior of the derivative $f_{u}(x, u)$. We omit the details of the proof (see [1] and [2] for similar results for the first two eigenvalues).

Theorem 3.3. Consider 3.20 with the following requirements:

(1) $f$ and $f_{u}$ are Carathéodory functions on $[0, L] \times \mathbb{R}$ and $f(\cdot, 0) \in L^{1}(0, L)$. 
(2) There exist $\alpha, \beta \in L^{\infty}(0, L)$ satisfying

$$
\lambda_{n} \leq \alpha(x) \leq f_{u}(x, u) \leq \beta(x)
$$

on $[0, L] \times \mathbb{R}$. Furthermore, $\alpha$ differs from $\lambda_{n}$ on a set of positive measure and $\beta$ satisfies either hypothesis (3.9) of Theorem 3.1 or hypothesis 3.14) of Theorem 3.2

Then problem (3.20) has a unique solution.

Acknowledgments. The authors have been supported by the Ministry of Education and Science of Spain (MTM2008-00988).

\section{References}

[1] Cañada, A., Montero, J. A., Villegas, S.: Liapunov-type inequalities and Neumann boundary value problems at resonance. Math. Inequal. Appl. 8, 459-475 (2005) Zbl 1085.34014 MR 2148238

[2] Cañada, A., Montero, J. A., Villegas, S.: Lyapunov inequalities for partial differential equations. J. Funct. Anal. 237, 176-193 (2006) Zbl pre05044037 MR 2239263

[3] Cañada, A., Villegas, S.: Optimal Lyapunov inequalities for disfocality and Neumann boundary conditions using $L^{p}$ norms. Discrete Contin. Dynam. Systems 20, 877-888 (2008) Zbl pre05347949 MR 2379477

[4] Dolph, C. L.: Nonlinear integral equations of Hammerstein type. Trans. Amer. Math. Soc. 66, 289-307 (1949) Zbl 0036.20202 MR 0032923

[5] Hartman, P.: Ordinary Differential Equations. Wiley, New York (1964) Zbl 0125.32102 MR 0171038

[6] Li, Y., Wang, H. Z.: Neumann problems for second order ordinary differential equations across resonance, Z. Angew. Math. Phys. 46, 393-406 (1995) Zbl 0824.34029 MR 1335909

[7] Lin, Y. H., Li, Y., Zhou, Q. D.: Second boundary value problems for nonlinear ordinary differential equations across resonance. Nonlinear Anal. 28, 999-1009 (1997) Zbl 0879.34066 MR 1422797

[8] Mawhin, J., Ward, J. R.: Nonuniform nonresonance conditions at the two first eigenvalues for periodic solutions of forced Liénard and Duffing equations. Rocky Mountain J. Math. 12, 643-654 (1982) Zbl 0536.34022 MR 0683859

[9] Mawhin, J., Ward, J. R.: Periodic solutions of some forced Liénard differential equations at resonance. Arch. Math. (Basel) 41, 337-351 (1983) Zbl 0537.34037| MR 0731606

[10] Mawhin, J., Ward, J. R.: Nonresonance and existence for nonlinear elliptic boundary value problems. Nonlinear Anal. 5, 677-684 (1981) Zbl 0475.35047 $\mid$ MR 0618221

[11] Mawhin, J., Ward, J. R., Willem, M.: Variational methods and semilinear elliptic equations. Arch. Ration. Mech. Anal. 95, 269-277 (1986) Zbl 0656.35044 MR 0853968

[12] Wang, H. Z., Li, Y.: Two-point boundary value problems for second order ordinary differential equations across many resonant points. J. Math. Anal. Appl. 179, 61-75 (1993) Zbl 0790.34025 MR 1244949

[13] Wang, H. Z., Li, Y.: Neumann boundary value problems for second-order ordinary differential equations across resonance. SIAM J. Control Optim. 33, 1312-1325 (1995) Zbl 0838.34028 MR 1348111

[14] Zhang, M.: Certain classes of potentials for $p$-Laplacian to be non-degenerate. Math. Nachr. 278, 1823-1836 (2005) Zbl 1092.34044 MR 2182093 